\title{
A New 3D Parallel Thinning Scheme Based on Critical Kernels
}

\author{
Gilles Bertrand and Michel Couprie \\ Institut Gaspard-Monge \\ Laboratoire A2SI, Groupe ESIEE \\ Cité Descartes, BP 99 \\ 93162 Noisy-le-Grand Cedex France \\ g.bertrand@esiee.fr, m.couprie@esiee.fr
}

\begin{abstract}
Critical kernels constitute a general framework settled in the category of abstract complexes for the study of parallel thinning in any dimension. We take advantage of the properties of this framework, and we derive a general methodology for designing parallel algorithms for skeletons of objects in 3D grids. In fact, this methodology does not need to handle the structure of abstract complexes, we show that only 3 masks defined in the classical cubic grid are sufficient to implement it. We illustrate our methodology by giving two new types of skeletons.
\end{abstract}

\section{Introduction}

Precisely forty years ago, in 1966, D. Rutovitz proposed an algorithm which is certainly the first parallel thinning algorithm [1. Since then, many 2D parallel thinning algorithms have been proposed, and several 3D ones may be found in the literature, see for example $[2,3,4,5,6,7,8,9,10,11,12$. A fundamental property required for such algorithms is that, they do preserve the topology of the original objects. In fact, such a guarantee is not obvious to obtain [13, 14. In [15], see also [16], one of the authors introduces a general framework for the study of parallel thinning in any dimension in the context of abstract complexes. A new definition of a simple point has been proposed, this definition is based on the collapse operation which is a classical tool in algebraic topology and which guarantees topology preservation. The most fundamental result is that, if a subset $Y$ of $X$ contains the so-called critical kernel of $X$, then $Y$ has the same topology as $X$.

In this paper, we focus on 3D objects. We introduce the notion of crucial voxels, which permits to make a link with the framework of digital topology [17. This leads to a general methodology for designing 3D parallel thinning algorithms. We illustrate our methodology by giving two new types of skeletons. The first one corresponds to a "minimal" skeleton of an object, the second one to a skeleton which contains a part of the medial axis of an object. All these skeletons are obtained by a sequence of symmetric operators. To our best knowledge, they have no equivalent. All previously proposed symmetric thinning conditions 
are not sufficiently "powerful" for removing enough points in order to obtain a skeleton such as the "minimal" skeleton of Fig. 7.

For the sake of space, proofs are not given in this paper, most of them will be available in an extended version of the paper.

\section{Cubical Complexes}

In this section, we give some basic definitions for cubical complexes, see also [18. We consider here the three-dimensional case. Note that many of the notions introduced in the first sections make sense in arbitrary $n$-dimensional cubical spaces.

If $T$ is a subset of $S$, we write $T \subseteq S$, we also write $T \subset S$ if $T \subseteq S$ and $T \neq S$.

Let $\mathbb{Z}$ be the set of integers. We consider the families of sets $\mathbb{F}_{0}^{1}, \mathbb{F}_{1}^{1}$, such that $\mathbb{F}_{0}^{1}=\{\{a\} \mid a \in \mathbb{Z}\}, \mathbb{F}_{1}^{1}=\{\{a, a+1\} \mid a \in \mathbb{Z}\}$. A subset $f$ of $\mathbb{Z}^{n}, n \geq 2$, which is the Cartesian product of exactly $m$ elements of $\mathbb{F}_{1}^{1}$ and $(n-m)$ elements of $\mathbb{F}_{0}^{1}$ is called a face or an $m$-face of $\mathbb{Z}^{n}, m$ is the dimension of $f$, we write $\operatorname{dim}(f)=m$.

We denote by $\mathbb{F}^{3}$ the set composed of all $m$-faces of $\mathbb{Z}^{3}, m=0,1,2,3$. An $m$-face of $\mathbb{Z}^{3}$ is called a point if $m=0$, a (unit) interval if $m=1$, a (unit) square if $m=2$, a (unit) cube if $m=3$.

Let $f$ be a face in $\mathbb{F}^{3}$. We set $\hat{f}=\left\{g \in \mathbb{F}^{3} \mid g \subseteq f\right\}$ and $\hat{f}^{*}=\hat{f} \backslash\{f\}$.

Any $g \in \hat{f}$ is a face of $f$, and any $g \in \hat{f}^{*}$ is a proper face of $f$.

If $X$ is a finite set of faces in $\mathbb{F}^{3}$, we write $X^{-}=\cup\{\hat{f} \mid f \in X\}, X^{-}$is the closure of $X$.

A set $X$ of faces in $\mathbb{F}^{3}$ is a cell or an $m$-cell if there exists an $m$-face $f \in X$, such that $X=\hat{f}$. The boundary of a cell $\hat{f}$ is the set $\hat{f}^{*}$.

A finite set $X$ of faces in $\mathbb{F}^{3}$ is a complex (in $\mathbb{F}^{3}$ ) if $X=X^{-}$. Any subset $Y$ of a complex $X$ which is also a complex is a subcomplex of $X$. If $Y$ is a subcomplex of $X$, we write $Y \preceq X$. If $X$ is a complex in $\mathbb{F}^{3}$, we also write $X \preceq \mathbb{F}^{3}$.

Let $X \preceq \mathbb{F}^{3}$. A face $f \in X$ is principal for $X$ if there is no $g \in X$ such that $f \in \hat{g}^{*}$. We denote by $X^{+}$the set composed of all principal faces of $X$.

Observe that, in general, $X^{+}$is not a complex, and that $\left[X^{+}\right]^{-}=X$. See illustrations Fig. 1 .

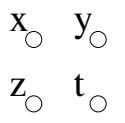

(a)

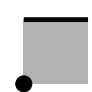

(b)

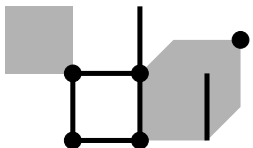

(c)

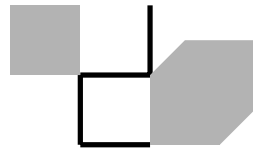

(d)

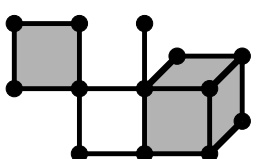

(e)

Fig. 1. (a): Four points $x, y, z, t$. (b): A graphical representation of the set of faces $\{\{x, y, z, t\},\{x, y\},\{z\}\}$. (c): A set of faces $X$, which is not a complex. (d): The set $X^{+}$, composed by the principal faces of $X$. (e): The set $X^{-}$, i.e. the closure of $X$, which is a complex. 
(a)

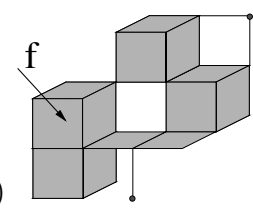

(b)

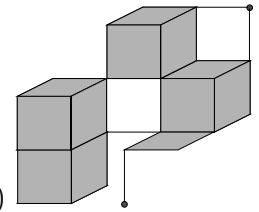

(c)

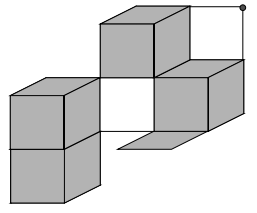

(d)

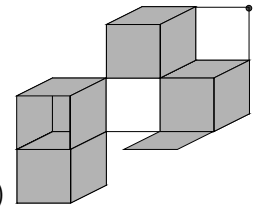

(e)

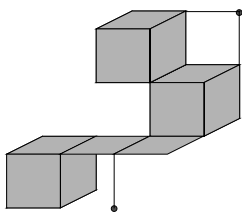

(f)

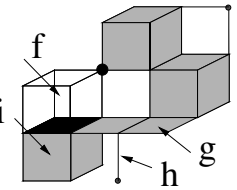

(g)

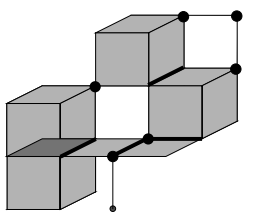

Fig. 2. (a) A complex $X$, (b), (c), and (d) three steps of elementary collapse of $X$, (e) the detachment of $\hat{f}$ from $X$, (f) the attachment of the 3-face $f$ is highlighted in dark, the face $f$ is not simple, whereas $g, h$, and $i$ are simple, (g) the essential faces of $X$ which are not principal are highlighted in dark

Let $X \preceq \mathbb{F}^{3}, \operatorname{dim}(X)=\max \left\{\operatorname{dim}(f) \mid f \in X^{+}\right\}$is the dimension of $X$. We say that $X$ is an $m$-complex if $\operatorname{dim}(X)=m$.

We say that $X$ is pure if, for each $f \in X^{+}$, we have $\operatorname{dim}(f)=\operatorname{dim}(X)$.

Let $X \preceq \mathbb{F}^{3}$ and $Y \preceq X$. If $Y^{+} \subseteq X^{+}$, we say that $Y$ is a principal subcomplex of $X$ and we write $Y \sqsubseteq X$. Observe that, for any $X \preceq \mathbb{F}^{3}, \emptyset \sqsubseteq X$.

If $X \preceq \mathbb{F}^{3}$ and if $X$ is a pure 3 -complex, we also write $X \sqsubseteq \mathbb{F}^{3}$.

Let $X \preceq \mathbb{F}^{3}$ and let $Y \preceq X$. We set $X \odot Y=\left[X^{+} \backslash Y^{+}\right]^{-}$. The set $X \odot Y$ is a complex which is the detachment of $Y$ from $X$.

\section{Simple Cells}

Intuitively a cell $\hat{f}$ of a complex $X$ is simple if its removal from $X$ "does not change the topology of $X$ ". In this section we propose a definition of a simple cell based on the operation of collapse [19, which is a discrete analogue of a continuous deformation (a homotopy). Note that this definition is a rather general one, in particular, it may be directly extended to $n$-dimensional cubical complexes [15.

Let $X$ be a complex in $\mathbb{F}^{3}$ and let $f \in X^{+}$. The face $f$ is a border face for $X$ if there exists one face $g \in \hat{f}^{*}$ such that $f$ is the only face of $X$ which contains $g$. Such a face $g$ is said to be free for $X$ and the pair $(f, g)$ is said to be a free pair for $X$. We say that $f \in X^{+}$is an interior face for $X$ if $f$ is not a border face.

Let $X$ be a complex, and let $(f, g)$ be a free pair for $X$. The complex $X \backslash\{f, g\}$ is an elementary collapse of $X$.

Let $X, Y$ be two complexes. We say that $X$ collapses onto $Y$ if there exists a collapse sequence from $X$ to $Y$, i.e., a sequence of complexes $\left\langle X_{0}, \ldots, X_{l}\right\rangle$ such that $X_{0}=X, X_{l}=Y$, and $X_{i}$ is an elementary collapse of $X_{i-1}, i=1, \ldots, l$. If $X$ collapses onto $Y$, we also say that $Y$ is a retraction of $X$. See illustration Fig. 2 (a), (b), (c), (d). 
We give now a definition of a simple point, it may be seen as a discrete analogue of the one given by T.Y. Kong in [20] which lies on continuous deformations in the $n$-dimensional Euclidean space.

Definition 1. Let $X \preceq \mathbb{F}^{3}$. Let $f \in X^{+}$.

We say that $\hat{f}$ and $f$ are simple for $X$ if $X$ collapses onto $X \odot \hat{f}$.

The notion of attachment, as introduced by T.Y. Kong [13, 20, leads to a local characterization of simple cells.

Definition 2. Let $X \preceq \mathbb{F}^{3}$ and let $f \in X^{+}$. The attachment of $\hat{f}$ for $X$ is the $\operatorname{complex} \operatorname{Attach}(\hat{f}, X)=\hat{f}^{*} \cap[X \odot \hat{f}]$.

In other words, a face $g$ is in $\operatorname{Attach}(\hat{f}, X)$ if $g$ is in $\hat{f}^{*}$ and if $g$ is a (proper) face of a principal face $h$ distinct from $f$.

The following proposition is an easy consequence of the above definitions.

Proposition 3. Let $X \preceq \mathbb{F}^{3}$, and let $f \in X^{+}$.

The cell $\hat{f}$ is simple for $\bar{X}$ if and only if $\hat{f}$ collapses onto $\operatorname{Attach}(\hat{f}, X)$.

The attachment of a 3-face $f$ of a complex $X$ is highlighted Fig. 2(f) and $X \odot \hat{f}$ is depicted in (e). It may be seen that $f$ is not simple: there is no collapse sequence from $X$ (a) to $X \otimes \hat{f}$ (e). On the other hand the faces $g, h$, and $i$ are simple.

\section{Critical Kernels}

Let $X$ be a complex in $\mathbb{F}^{3}$. We observe that, if we remove simultaneously simple cells from $X$, we may obtain a set $Y$ such that $X$ does not collapse onto $Y$. In other words, if we remove simple cells in parallel, we may "change the topology" of the original object $X$. Thus, it is not possible to use directly the notion of simple cell for thinning discrete objects in a symmetrical manner.

In this section, we introduce a framework for thinning in parallel discrete objects with the warranty that we do not alter the topology of these objects. This method may be extended for complexes of arbitrary dimension [15. As far as we know, this is the first method which allows to thin arbitrary complexes in a symmetric way.

This method is based solely on three notions, the notion of an essential face which allows to define the core of a face, and the notion of a critical face.

Definition 4. Let $X \preceq \mathbb{F}^{3}$ and let $f \in X$. We say that $f$ is an essential face for $X$ if $f$ is precisely the intersection of all principal faces of $X$ which contain $f$, i.e., if $f=\cap\left\{g \in X^{+} \mid f \subseteq g\right\}$. We denote by $E s s(X)$ the set composed of all essential faces of $X$. If $f$ is an essential face for $X$, we say that $\hat{f}$ is an essential cell for $X$.

Observe that a principal face for $X$ is necessarily an essential face for $X$, i.e., $X^{+} \subseteq \operatorname{Ess}(X)$. The essential and non-principal faces of the complex $X$ of Fig. 2 (a) are highlighted Fig. 2 (g). 


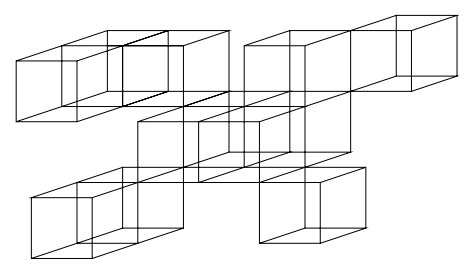

(a)

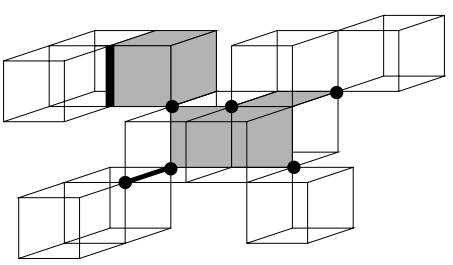

(b)

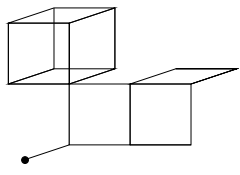

(c)

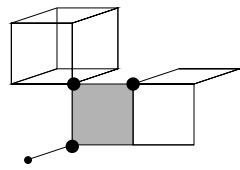

(d)

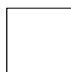

(e)

Fig. 3. (a): A complex $X_{0}$ in $\mathbb{F}^{3}$. (b): The critical faces of $X_{0}$ are highlighted. (c) The complex $X_{1}=\operatorname{Critic}\left(X_{0}\right)$. (d): The critical faces of $X_{1}$ are highlighted. (e) The complex $X_{2}=\operatorname{Critic}\left(X_{1}\right): X_{2}$ is such that $\operatorname{Critic}\left(X_{2}\right)=X_{2}$.

Definition 5. Let $X \preceq \mathbb{F}^{3}$ and let $f \in \operatorname{Ess}(X)$. The core of $\hat{f}$ for $X$ is the complex, denoted by $\operatorname{Core}(\hat{f}, X)$, which is the union of all essential cells for $X$ which are in $\hat{f}^{*}$, i.e., Core $(\hat{f}, X)=\cup\left\{\hat{g} \mid g \in \operatorname{Ess}(X) \cap \hat{f}^{*}\right\}$.

The preceding definition may be seen as a generalization of the notion of attachment for arbitrary essential cells (not necessarily principal).

Proposition 6. Let $X \preceq \mathbb{F}^{3}$ and let $f \in X^{+}$. The attachment of $\hat{f}$ for $X$ is precisely the core of $\hat{f}$ for $X$, i.e, we have $\operatorname{Attach}(\hat{f}, X)=\operatorname{Core}(\hat{f}, X)$.

Definition 7. Let $X \preceq \mathbb{F}^{3}$ and let $f \in X$. We say that $f$ and $\hat{f}$ are regular for $X$ if $f \in \operatorname{Ess}(X)$ and if $\hat{f}$ collapses onto $\operatorname{Core}(\hat{f}, X)$. We say that $f$ and $\hat{f}$ are critical for $X$ if $f \in \operatorname{Ess}(X)$ and if $f$ is not regular for $X$.

We set $\operatorname{Critic}(X)=\cup\{\hat{f} \mid f$ is critical for $X\}, \operatorname{Critic}(X)$ is the critical kernel of $X$. A face $f$ in $X$ is a maximal critical face, or an $M$-critical face for $X$, if $f$ is a principal face of $\operatorname{Critic}(X)$.

Again, the preceding definition of a regular cell is a generalization of the notion of a simple cell. As a corollary of Prop. 6, a principal face of a complex $X \preceq \mathbb{F}^{3}$ is regular for $X$ if and only if is simple for $X$.

The following theorem holds for complexes of arbitrary dimensions (see [15]). This is our basic result in this framework. See Fig. 3 where the successive critical kernels of a complex are depicted.

Theorem 8. Let $X \preceq \mathbb{F}^{3}$. The critical kernel of $X$ is a retraction of $X$. Furthermore, if $Y \sqsubseteq X$ is such that $Y$ contains the critical kernel of $X$, then $Y$ is a retraction of $X$. 


\section{Crucial Kernels}

If $X$ is a complex in $\mathbb{F}^{3}$, the subcomplex $\operatorname{Critic}(X)$ is not necessarily a principal subcomplex of $X$ as illustrated Fig. 3. In this paper, we investigate thinning algorithms which take as input a pure 3-complex and which return a principal subcomplex of the input (thus also a pure 3-complex). For that purpose, we propose some notions which allow to recover a principal subcomplex $Y$ of a pure complex $X$, with the constraint that $Y$ is a retraction of $X$.

Definition 9. Let $X \sqsubseteq \mathbb{F}^{3}$, and let $f \in X^{+}$be a 3-face for $X$.

We say that $f$ and $\overline{\hat{f}}$ are 3 -crucial for $X$ if $f$ is critical for $X$. We say that $f$ and $\hat{f}$ are $k$-crucial for $X, k=2,1,0$, if $\hat{f}^{*}$ contains a $k$-face which is M-critical for $X$ and which is not a proper face of an $l$-crucial face, $l>k$. We say that $f$ and $\hat{f}$ are crucial for $X$ if $f$ is $k$-crucial for some $k \in[0,1,2,3]$.

Thus, a 3 -face $f$ is 2 -crucial iff it contains a 2 -face which is M-critical. A 3 -face $f$ is 1-crucial iff it contains a 1-face which is M-critical and which is not contained in a 2-crucial face. A 3 -face $f$ is 0 -crucial iff it contains a 0 -face which is $\mathrm{M}$ critical and which is not contained in a 2 - or 1-crucial face. Observe that a face $f$ which is $k$-crucial cannot be $l$-crucial, with $l \neq k$.

In Fig. 4 (a), the M-critical faces of a complex are highlighted (see also Fig. 3 (b) where the critical faces of the same complex are given). The 3-face $h$ is not crucial: it contains a 0 -face which is critical but not M-critical. The 3 -face $f$ is 3 -crucial, the 3 -face $g$ is 2 -crucial. The 3 -face $i$ is not crucial: it contains a 1 -face which is M-critical but which is a face of the 2-crucial face $g$.

Let $X \sqsubseteq \mathbb{F}^{3}$. We define the crucial kernel of $X$ as the set $\operatorname{Cruc}(X)$ which is the union of all cells of $X$ which are crucial for $X$. By the very definition of a crucial face, $\operatorname{Cruc}(X)$ contains the critical kernel of $X$. Thus, by Prop. 8 , the crucial kernel of $X$ is a retraction of $X$.

In Fig. 4 (a), a complex $X_{0}$ and its M-critical faces are depicted (5 faces). The complex $X_{1}=\operatorname{Cruc}\left(X_{0}\right)$ is given in (b) also with its M-critical faces (only one 2 -face). Finally, in (c), the complex $X_{2}=\operatorname{Cruc}\left(X_{1}\right)$ contains also one M-critical face, and it may be seen that $X_{2}=\operatorname{Cruc}\left(X_{2}\right)$.

For thinning objects, we often want to keep other faces than the ones which are crucial. That is why we introduce the following definition in order to generalize the previous notions. Intuitively, the set $K$ corresponds to a set which is preserved by a thinning algorithm (like extremities of curves, if we want to obtain a curvilinear skeleton).

Definition 10. Let $X \sqsubseteq \mathbb{F}^{3}$, let $K \subseteq X^{+}$be a set composed of 3-faces of $X$, and let $f \in X^{+}$be a 3 -face of $X$.

We say that $f$ and $\hat{f}$ are 3 -crucial for $\langle X, K\rangle$ if $f$ is critical for $X$ or if $f$ is in $K$. We say that $f$ and $\hat{f}$ are $k$-crucial for $\langle X, K\rangle, k=2,1,0$, if $\hat{f}^{*}$ contains a $k$-face $g$ which is M-critical for $X$ and which is not a proper face of an $l$-crucial face for $\langle X, K\rangle$, with $l>k$. The set of 3-faces of $X$ which contain such a face $g$ is a $k$-crucial clique for $\langle X, K\rangle$. 


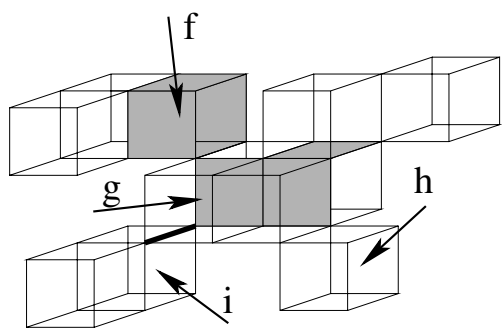

(a)

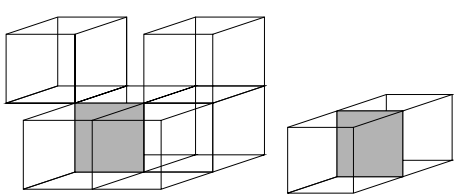

(b)

(c)

Fig. 4. (a): A complex $X_{0}$ and its M-critical faces (highlighted). (b): $X_{1}=\operatorname{Cruc}\left(X_{0}\right)$ and its M-critical faces. (c): The complex $X_{2}=\operatorname{Cruc}\left(X_{1}\right)$ contains only one M-critical face (highlighted), and $X_{2}=\operatorname{Cruc}\left(X_{2}\right)$.

We say that $f$ and $\hat{f}$ are crucial for $\langle X, K\rangle$ if, for some $k \in[0,1,2,3], f$ is $k$-crucial for $\langle X, K\rangle$. We say that a set of 3-faces is a crucial clique for $\langle X, K\rangle$ if, for some $k \in[0,1,2]$, this set is a a $k$-crucial clique for $\langle X, K\rangle$.

Definition 11. Let $X \sqsubseteq \mathbb{F}^{3}$, and let $K \subseteq X^{+}$be a set composed of 3-faces of $X$. The crucial kernel of $X$ constrained by $K$ is the set which is the union of all crucial cells for $\langle X, K\rangle$.

From the previous definitions and from Th. 8, we immediately deduce the following proposition which ensures that any constrained crucial kernel of an object preserves the topology of this object.

Proposition 12. Let $X \sqsubseteq \mathbb{F}_{2}^{n}$, and let $K \subseteq X^{+}$be a set composed of 3-faces of $X$. The crucial kernel of $X$ constrained by $K$ is a retraction of $X$.

\section{Crucial Voxels in the Cubic Grid}

We introduce the following definitions in order to establish a link between pure complexes in $\mathbb{F}^{3}$ and objects in the cubic grid as often considered in image processing.

We define the cubic grid as the set $\mathbb{G}^{3}$ composed of all 3 -faces of $\mathbb{F}^{3}$. A 3-face of $\mathbb{G}^{3}$ is also called a voxel. In the sequel, we consider only finite subsets of $\mathbb{G}^{3}$.

For any pure complex in $\mathbb{F}^{3}$, i.e., for any $X \sqsubseteq \mathbb{F}^{3}$, we associate the subset $X^{+}$of $\mathbb{G}^{3}$. In return, to each finite subset $S$ of $\mathbb{G}^{3}$, we associate the complex $S^{-}$of $\mathbb{F}^{3}$. This will be our basic methodology to "interpret" a set of voxels. In particular, all definitions given for a principal face in $X^{+}$have their counterparts for a voxel in $\mathbb{G}^{3}$. For example if $S \subseteq \mathbb{G}^{3}$ and $p \in S$, we will say that the voxel $p$ is simple for $S$ if $p$ is simple for $S^{-}$. Border, interior, $(k-)$ critical, and $(k-)$ crucial voxels are defined in the same manner.

Some local characterizations of simple voxels in the cubic grid have been proposed [21,22, 23, these characterizations may be used for detecting 3-crucial voxels (i.e., non-simple voxels). We give now some simple local conditions, also 


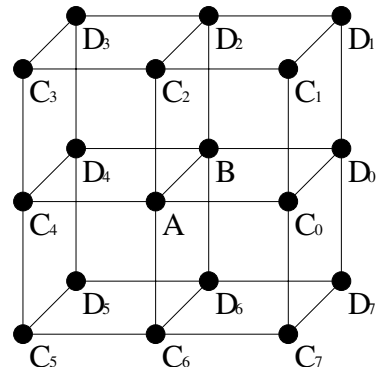

$M_{2}$

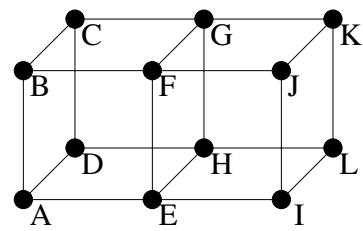

$M_{1}$

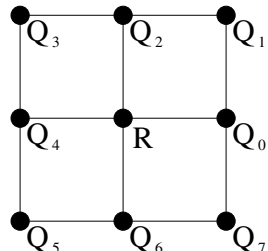

$M_{2}^{\prime}$

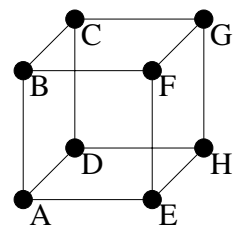

$M_{0}$

Fig. 5. Masks for 2-crucial $\left(M_{2}\right)$, 1-crucial $\left(M_{1}\right)$, and 0 -crucial $\left(M_{0}\right)$ voxels, $M_{2}^{\prime}$ is a configuration derived from $M_{2}$. Here, a voxel is represented by a point.

in the cubic grid, for $k$-crucial voxels, with $k=2,1,0$. We express these local conditions by a set of masks, as in most papers related to parallel thinning in the digital topology framework. These masks $M_{2}, M_{1}, M_{0}$ are given Fig. 5] For each of these masks, we also consider all the masks obtained from them by applying $\pi / 2$ rotations. We get 7 masks ( 3 for $M_{2}, 3$ for $M_{1}$, and 1 for $M_{0}$ ). The $2 \mathrm{D}$ configuration $M_{2}^{\prime}$, which appears also in Fig. 5, is derived from $M_{2}$ as explained in the following. Different characterizations for 2D simple configurations may be found in [17, they may be used for checking condition iii) for $M_{2}^{\prime}$. See also Fig. 6 for an illustration of the use of mask $M_{2}$.

Definition 13. Let $S \subseteq \mathbb{G}^{3}$, and let $M$ be a set of voxels of $S$.

1) The set $M$ matches the mask $M_{2}$ if:

i) the voxels in $M$ are simple for $S$; and

ii) $M=\{A, B\}$; and

iii) the $2 \mathrm{D}$ configuration $M_{2}^{\prime}$ obtained by setting $R \in M_{2}^{\prime}$ and setting $Q_{i} \in M_{2}^{\prime}$ if $\left\{C_{i}, D_{i}\right\} \cap S \neq \emptyset$, with $i \in[0, \ldots, 7]$, is such that $R$ is non-simple in the 2D sense.

2) The set $M$ matches the mask $M_{1}$ if:

i) the voxels in $M$ are simple and not 2-crucial for $S$; and

ii) $M=\{E, F, G, H\} \cap S$; and

iii) the set $\{E, G\}$ or the set $\{F, H\}$ (or both) is included in $M$; and

iv) we have either $[U \cap S \neq \emptyset$ and $V \cap S \neq \emptyset]$ or $[U \cap S=\emptyset$ and $V \cap S=\emptyset$ ], with $U=\{A, B, C, D\}$ and $V=\{I, J, K, L\}$.

3) The set $M$ matches the mask $M_{0}$ if:

i) the voxels in $M$ are simple and neither 2-crucial nor 1-crucial for $S$; and 


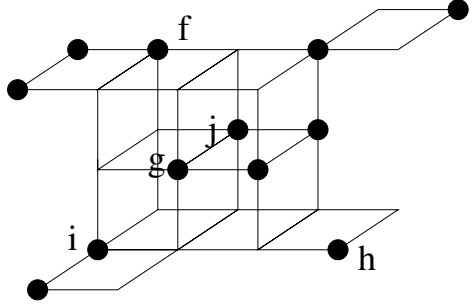

(a)

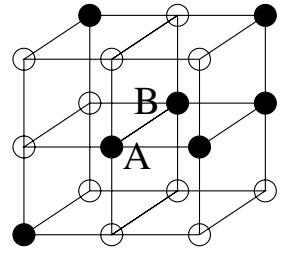

(b)

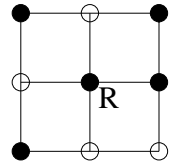

(c)

Fig. 6. (a): The subset $S$ of $\mathbb{G}^{3}$ which corresponds to the complex $X_{0}$ of Fig. 4 (a). Each voxel of $S$ is represented by a black disk. (b): The mask $M_{2}$, with $A, B$ matching voxels $g, j$ of $S$. (c): The corresponding configuration of mask $M_{2}^{\prime}$. The element $R$ is not simple in the $2 \mathrm{D}$ sense, thus the voxels $g, j$ of $S$ constitute a 2 -crucial clique.

ii) $M=\{A, B, C, D, E, F, G, H\} \cap S$; and

iii) at least one of the sets $\{A, G\},\{B, H\},\{C, E\},\{D, F\}$ is a subset of $M$.

Proposition 14. Let $S \subseteq \mathbb{G}^{3}, K \subseteq S$, and let $M$ be a set of voxels in $S \backslash K$. i) $M$ is a 2-crucial clique for $\langle X, K\rangle$ if and only if $M$ matches the mask $M_{2}$; ii) $M$ is a 1-crucial clique for $\langle X, K\rangle$ if and only if $M$ matches the mask $M_{1}$; iii) $M$ is a 0-crucial clique for $\langle X, K\rangle$ if and only if $M$ matches the mask $M_{0}$.

Observe that a voxel is $k$-crucial iff it belongs to a $k$-crucial clique, with $k \in$ $[0,1,2]$. Thus, Prop. 14 also provides a method for detecting 0-, 1-, and 2-crucial voxels.

\section{A Generic Thinning Scheme}

We define the following notion of skeleton which is constrained to include a given set $K$. We then give an algorithm for computing this skeleton, this algorithm may be viewed as a generic thinning scheme where different kinds of skeletons may be obtained by considering different sets $K$. At last, we give two examples of skeletons derived from this scheme: one is a minimal skeleton, the other is constrained to contain some of the centers of the maximal balls included in the object. All these skeletons are obtained by a sequence of symmetric operators, thus they are invariant by $\pi / 2$ rotations.

Definition 15. Let $S \subseteq \mathbb{G}^{3}$ and let $K \subseteq S$. We denote by $\operatorname{Cruc}(S, K)$ the set composed of all voxels which are crucial for $\langle S, K\rangle$.

Let $\left\langle S_{0}, S_{1}, \ldots, S_{k}\right\rangle$ be the unique sequence such that $S_{0}=S, S_{k}=\operatorname{Cruc}\left(S_{k}, K\right)$ and $S_{i}=\operatorname{Cruc}\left(S_{i-1}, K\right), i=1, \ldots, k$. The set $S_{k}$ is the $\mathcal{K}$-skeleton of $S$ constrained by $K$.

By Prop. 12, the $\mathcal{K}$-skeleton of a set $S$ constrained by a set $K$ is a retraction of $S$. By construction, the following algorithm computes the $\mathcal{K}$-skeleton of $S$ constrained by $K$. It consists in the repetition of 5 steps, each step may be done 


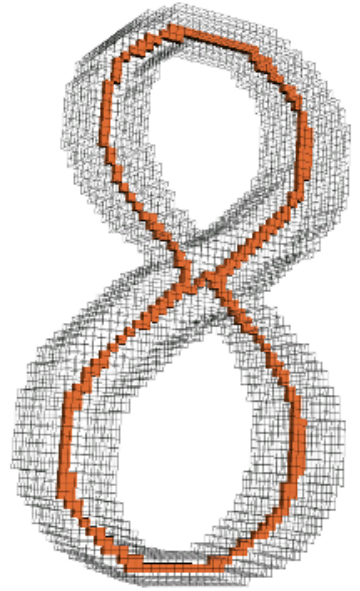

(a)

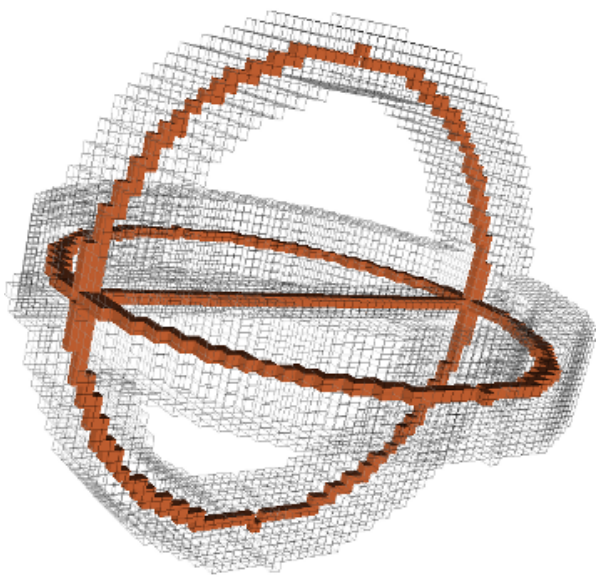

(b)

Fig. 7. Two objects in $\mathbb{G}^{3}$ and their minimal $\mathcal{K}$-skeleton (in red)

in parallel, with the local characterization of critical (non-simple) voxels (step 02), and with the characterizations given Prop. 14 (steps 03, 04, 05).

$S K^{3}$ (Input: $S \subseteq \mathbb{G}^{3}, K \subseteq S$; Output: $S$ )

01. Repeat Until Stability

02. $\quad R_{3}:=$ set of voxels which are critical for $S$ or which are in $K$

03. $\quad R_{2}:=$ set of voxels which belong to a 2-crucial clique included in $S \backslash R_{3}$

04. $\quad R_{1}:=$ set of voxels which belong to a 1-crucial clique included in $S \backslash\left(R_{3} \cup R_{2}\right)$

05. $\quad R_{0}:=$ set of voxels which belong to a 0 -crucial clique included in $S \backslash\left(R_{3} \cup R_{2} \cup R_{1}\right)$

06. $\quad S:=R_{3} \cup R_{2} \cup R_{1} \cup R_{0}$

A minimal skeleton of an object may be obtained by imposing no constraining set. Let $S \subseteq \mathbb{G}^{3}$. The minimal $\mathcal{K}$-skeleton of $S$ is defined as the $\mathcal{K}$-skeleton of $S$ constrained by $K$, with $K=\emptyset$.

Two examples of minimal $\mathcal{K}$-skeletons are given Fig. 7 As far as we know, $S K^{3}$ is the first thinning scheme which allows to compute such skeletons which are invariant by $\pi / 2$ rotations. Furthermore, the result of $S K^{3}$ is an object which is well-defined. To our best knowledge, this is also the first attempt to give a precise definition of such a notion.

The quality of a skeleton is often assessed by the fact that it contains, approximately or completely, the medial axis of the shape.

Let $S \subseteq \mathbb{G}^{3}$. We consider the balls induced by the city-block distance. A ball is maximal for $S$ if it is included in $S$ and if it is not a proper subset of another ball included in $S$. We denote by $K_{r}$ the set composed of the centers of all maximal balls which have a radius greater than or equal to $r$. The medial axis of $S$ is precisely the set $K_{0}$. In [24], A. Rosenfeld and J.L. Pfaltz have proved that, for the city-block and the chessboard distance, the medial axis of a shape can be obtained by detecting the local maxima of its distance transform. This provides an algorithm for computing any set $K_{r}$. In Fig. 8, different $\mathcal{K}$-skeletons constrained 


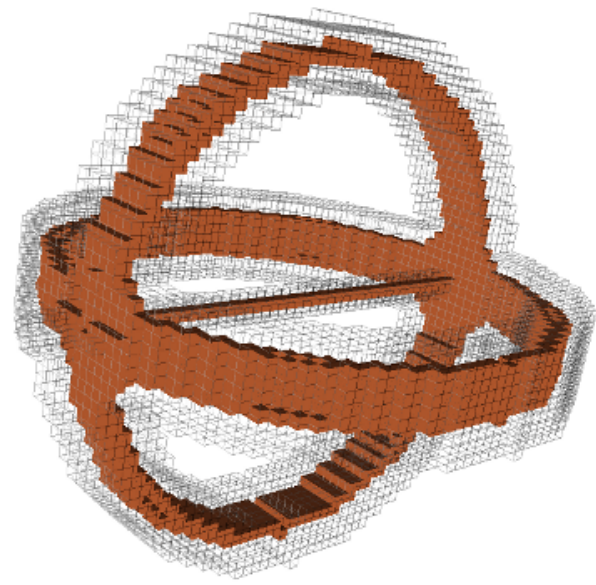

(a)

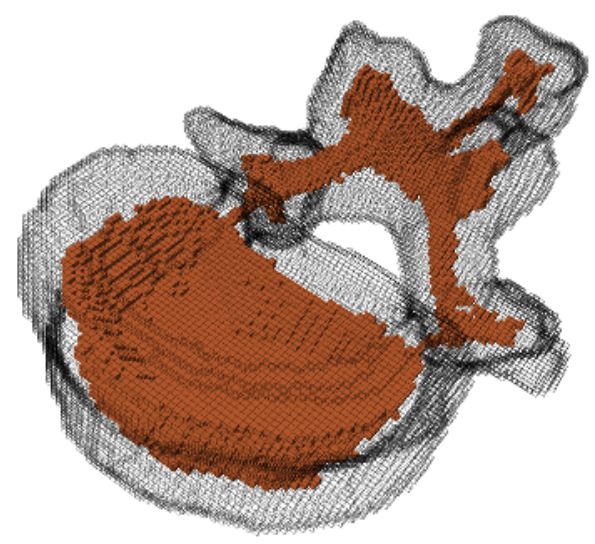

(c)

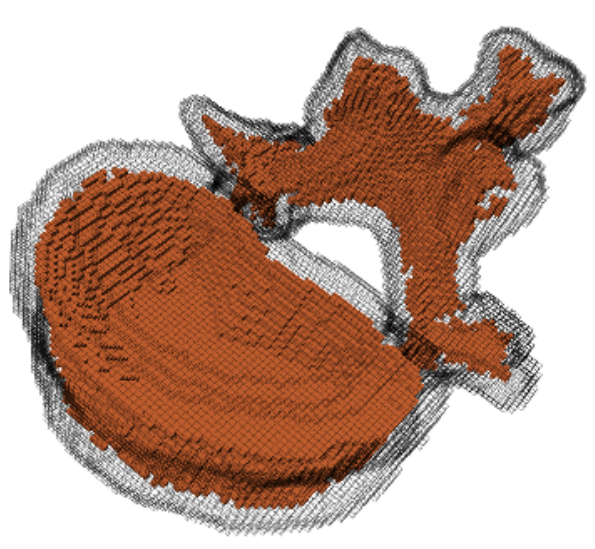

(b)

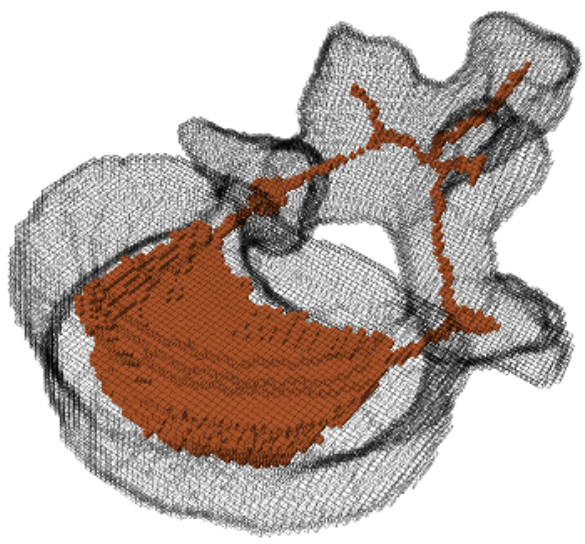

(d)

Fig. 8. (a): A subset $S$ of $\mathbb{G}^{3}$ and its $\mathcal{K}$-skeleton constrained by $K_{3}$. (b): A subset $T$ of $\mathbb{G}^{3}$ and its $\mathcal{K}$-skeleton constrained by $K_{5}$. (c): The $\mathcal{K}$-skeleton of $T$ constrained by $K_{7}$. (d): The $\mathcal{K}$-skeleton of $T$ constrained by $K_{9}$.

by $K_{r}$ are given. As far as we know, this is the first thinning method which allows to obtain such skeletons constrained by a set and which are invariant by $\pi / 2$ rotations.

\section{References}

1. Rutovitz, D.: Pattern recognition. Journal of the Royal Statistical Society $\mathbf{1 2 9}$ (1966) 504-530

2. Tsao, Y., Fu, K.: A parallel thinning algorithm for 3d pictures. CGIP 17(4) (1981) 315-331 
3. Saha, P., Chaudhuri, B., Dutta, D., Majumder, D.: A new shape-preserving parallel thinning algorithm for 3d digital images. PR 30(12) (1997) 1939-1955

4. Bertrand, G.: A parallel thinning algorithm for medial surfaces. PRL 16 (1995) 979-986

5. Gong, W., Bertrand, G.: A simple parallel 3d thinning algorithm. In: ICPR90. (1990) 188-190

6. Ma, C.M.: A 3d fully parallel thinning algorithm for generating medial faces. Pattern Recogn. Lett. 16(1) (1995) 83-87 FILE=ma1995.pdf.

7. Manzanera, A., Bernard, T., Prêteux, F., Longuet, B.: N-dimensional skeletonization: a unified mathematical framework. Journal of Electronic Imaging 11(1) (2002) 25-37

8. Ma, C.M., Sonka, M.: A 3d fully parallel thinning algorithm and its applications. Computer Vision and Image Understanding 64(3) (1996) 420-433

9. Palágyi, K., Kuba, A.: A 3d 6-subiteration thinning algorithm for extracting medial lines. Pattern Recognition Letters (19) (1998) 613-627

10. Palágyi, K., Kuba, A.: A parallel 3d 12-subiteration thinning algorithm. Graphical Models and Image Processing (61) (1999) 199-221

11. Lohou, C., Bertrand, G.: A 3d 12-subiteration thinning algorithm based on psimple points. Discrete Applied Mathematics 139 (2004) 171-195

12. Lohou, C., Bertrand, G.: A 3d 6-subiteration curve thinning algorithm based on p-simple points. Discrete Applied Mathematics 151 (2005) 198-228

13. Kong, T.Y.: On topology preservation in 2-d and 3-d thinning. International Journal on Pattern Recognition and Artificial Intelligence 9 (1995) 813-844

14. Bertrand, G.: On P-simple points. Comptes Rendus de l'Académie des Sciences, Série Math. I(321) (1995) 1077-1084

15. Bertrand, G.: On critical kernels. Internal Report, Université de Marne-la-Vallée IGM2005-05 (2005) Also submitted for publication.

16. Bertrand, G., Couprie, M.: Two-dimensional parallel thinning algorithms based on critical kernels. Internal Report, Université de Marne-la-Vallée IGM2006-02 (2006) Also submitted for publication.

17. Kong, T.Y., Rosenfeld, A.: Digital topology: introduction and survey. Comp. Vision, Graphics and Image Proc. 48 (1989) 357-393

18. Kovalevsky, V.: Finite topology as applied to image analysis. Computer Vision, Graphics and Image Processing 46 (1989) 141-161

19. Giblin, P.: Graphs, surfaces and homology. Chapman and Hall (1981)

20. Kong, T.Y.: Topology-preserving deletion of 1's from 2-, 3- and 4-dimensional binary images. In: Lecture Notes in Computer Science. Volume 1347. (1997) 3-18

21. Bertrand, G.: Simple points, topological numbers and geodesic neighborhoods in cubic grids. Pattern Recognition Letters 15 (1994) 1003-1011

22. Bertrand, G., Malandain, G.: A new characterization of threedimensional simple points. Pattern Recognition Letters 15(2) (1994) 169-175

23. Saha, P., Chaudhuri, B., Chanda, B., Dutta Majumder, D.: Topology preservation in 3d digital space. PR 27 (1994) 295-300

24. Rosenfeld, A., Pfaltz, J.: Sequential operations in digital picture processing. Journal of the Association for Computer Machinery 13 (1966) 471-494 